\title{
Atrial Conduction Time measured preoperatively By Tissue Doppler as a Predictor of Atrial Fibrillation after Mitral Valve Surgery for Mitral Regurge Patients
}

H.H.Ebaid , A.M.Ahmed and Kh.A.Al Rabat

Cardiology Dept., Faculty of Medicine, Benha Univ., Benha, Egypt

E-Mail:mahamoda@gmail.com

\begin{abstract}
Atrial fibrillation (AF) is one of the common adverse effects of cardiac surgery with an incidence of approximately $65 \%$. The present study aimed to evaluate the value PA-TDI duration ability to predict post-operative AF. Data of 100 patients who was diagnosed with severe mitral regurge and was referred for mitral valve surgery. PA interval was measured using tissue Doppler as well as routine echocardiographic parameters. After surgery patients was monitored to detect any attacks of atrial fibrillation. According to occurrence of atrial fibrillation patients was divided to two groups and compared the two groups. PA-TDI interval duration in group I was significantly prolonged than in group II P-value $<0.001$. PA-TDI duration more than $151 \mathrm{~ms}$ had an accuracy of $66 \%$ in detecting AF episodes post- operatively with sensitivity equal to 65 and specificity $68 \%$. Positive predictive value for PA-TDI interval more than $151 \mathrm{~ms}$ was $76 \%$ and negative predictive value of this interval was $53 \%$. This study discovered that Pre-operative PA-TDI duration represent an independent predictor of POAF. PA-TDI is a simple parameter whose cost is low, is not a time-consuming parameter to be measured, and is available in many modern echocardiographic.
\end{abstract}

Keywords: post-operative atrial fibrillation , mitral regurge, TDI-PA interval, Total atrial conduction time.

\section{Introduction}

Atrial fibrillation (AF) is one of the common adverse effects of cardiac surgery with an incidence of approximately $65 \%$ [1]. Current developments in surgical techniques and preand post-operative care allow high-risk patients to be able to undergo cardiac surgery. In addition, recent development in cardiac rhythm monitoring helped to detect AF episodes with higher accuracy and sensitivity [2].

However, old studies considered postoperative $\mathrm{AF}$ as benign, self-limiting complication or side effect of cardiac surgery which has no consequence [1], recent studies stated various adverse effects such as prolonged length of admission, cerebral infraction, the need of a permanent pacemaker and short- and long-term mortality [3].

However, many of the previous studies focused on long-term morbidity and mortality, this study focusses on predicting post-operative atrial fibrillation to help the physician and the patient to obtain the appropriate treatment options for those patients [4].

Some specific predictors can be used for detecting patients at risk for post-operative AF. Some of these is predictors have been investigated but with a little or limited value. PA-TDI has been investigated in many clinical scenarios regarding post-operative AF prediction after aortic valve intervention and $\mathrm{CABG}$; however, there is limited data about the association of PA-TDI and POAF after mitral valve surgical intervention [5].

\section{Patients and methods}

Among patients with valvular affection who underwent mitral valve surgical intervention, 100 patients with no clinical history of atrial arrhythmias one week before surgery were chosen. Patients with implanted pacemaker or those who are on therapy for arrhythmia except B-blockers were excluded from the study.

The same medications and B-blockers were resumed before and after the surgery. Old patients above the age of 70 were excluded from this study to exclude the age role in $\mathrm{AF}$ development. Preoperatively, the presence of risk factors such as diabetes, hypertension, and smoking history was obtained from the patients.

Echocardiography was used to detect Ejection fraction, regional wall motion abnormality and mitral regurgitation. Angiography was done to all patients to exclude or confirm CAD. A 12-lead ECG was preoperatively recorded at a voltage of 10 $\mathrm{mm} / \mathrm{mV}$ and speeds of $25 \mathrm{~mm} / \mathrm{s}$ one day or days before operation.

The same standard was fixed for all ECGs. On surface ECG, P-wave duration was measured from the START (connection between the isoelectric line and the START of $\mathrm{P}$-wave deflection) and the end of the P-wave.

PWD was calculated by measuring the difference between the duration of the longest and the shortest P-waves in lead II. Postoperative AF was detected by monitoring the patient during hospital admission. The primary 
end point, AF, was defined as irregular rhythm with duration longer than 30 seconds.

The differences between pre- and postoperative parameters were evaluated by Fisher's exact test. $\mathrm{P}$ values lower than 0.05 were considered significant. Mann- Whitney test was done for comparison between variables pre and post-surgery. Linear regression analysis to search for associations between parameters pre and post valve replacement. Statistical analysis was done by SPSS (SPSS Inc., Chicago, IL, US).

\section{Results}

\subsection{Patient characteristics}

One hundred patients undergoing mitral valve surgical intervention were enrolled in the study.

The clinical date of this study patients is summarized in Table (1).

The age of the patients in our study was 66 \pm 10 years of age, and $43 \%$ were women. All patients group, 100 patients $(76 \%)$ underwent mitral valve replacement by metallic valve, 25 patients $(25 \%)$ tissue valve, and 10 patients (10\%) underwent combined mitral valve replacement and tricuspid valve repair.

Sixty-six (66\%) had an attack or more of AF within hospital admission after cardiac surgery before discharge. There was relation between the risk of POAF and drugs prescribed to the patient prior to surgery and his co-morbidities.

Patients with POAF had no significant difference from the sinus group $(68.6 \pm 2.1 \mathrm{vs}$ $64.4 \pm 1.6$ years; $\mathrm{P}=0.12$ ).

Regarding baseline characteristics, there was no statistically significant difference among patients who underwent right atrial tissue sample taking and those who did not have their right atrium appendage sampled $(\mathrm{P}=$ $0.05)$.

\subsection{Echocardiographic parameters of POAF and the importance of TACT}

Comparisons of date collected by echocardiography in patients with POAF and patient who remained in sinus rhythm are summarized in Table (2).

Mean left atrium diameter was relatively the same in patients with POAF and no POAF $(42.4 \pm 0.97$ vs $40.3 \pm 0.95$ milliseconds; $\mathrm{P}=$ 0.12 ), left atrium volume indexed to body surface area was also the same in both groups $(32.8 \pm 2.4$ vs $27.1 \pm 1.7 \mathrm{~mL} / \mathrm{m} 2 ; \mathrm{P}=0.06)$. Moreover, left atrial ejection fraction was significantly lower in patients who developed POAF in comparison with NO POAF group $(45.6 \pm 2.1$ vs $54.0 \pm 2.7 \% ; \mathrm{P}=0.02)$.

The mean PA-TDI duration for the total study population was 132.8 milliseconds with a range from 87 to 185 milliseconds (mean coefficient of variation: $1.9 \pm 1.74 \%$ ).

Patients with POAF during the first 10 days after cardiac surgery had a significantly longer PA-TDI interval at baseline compared to patients who maintained in sinus rhythm (152.1 \pm 3.0 vs $120.8 \pm 1.8$ milliseconds; mean \pm standard error of the mean [SEM]; P < 0.001). At an optimal cut-off value of 133 milliseconds, the sensitivity and specificity in identifying POAF were $100 \%$ and $86 \%$, respectively (Fig. $3)$. On multivariate logistic regression analysis, PA-TDI interval (milliseconds), LA-EF (\%) and LAVI $(\mathrm{mL} / \mathrm{m} 2)$ were independently associated with POAF $(\mathrm{P}<0.05)$ Table (3)

Table (1) Clinical parameters of the Study Population.

\begin{tabular}{lccc}
\hline & POAF $(\mathbf{n = 6 6})$ & No POAF $(\mathbf{n}=\mathbf{4 4})$ & P value \\
\hline Age & $64.4 \pm 1.6$ & $68.6 \pm 2.1$ & 0.12 \\
Sex & $\mathrm{m}: 39$ & $\mathrm{~m}: 28$ & 0.74 \\
Body surface area (m2) & $2.0+-.1$ & $2.0+-.1$ & 0.12 \\
Body mass index (kg/m2) & $28.5 \pm 0.6$ & $26.4 \pm 0.8$ & 0.74 \\
CHA2DS2VASc-Score & $3(2)$ & $3(2.5)$ & 0.96 \\
Underlying diseases (n) & & & \\
Hypertension & 12 & 18 & 0.30 \\
Diabetes mellitus & 4 & 8 & 0.74 \\
History of stroke or TIA & 1 & 3 & 0.82 \\
Chronic kidney disease & 1 & 2 & 0.69 \\
smoking & 24 & 12 & 0.42 \\
B-blocking agents & 30 & 19 & 0.74 \\
Verapamil & 5 & 8 & 0.97 \\
Statins & 47 & 22 & 0.48 \\
Angiotensin-converting enzyme inhibitor & 44 & 31 & 0.86 \\
Number of antihypertensive medications & 2 & 2 & 0.44 \\
\hline
\end{tabular}

Data are expressed as Means \pm SEM and count respectively. 
Table (2) Characteristics of the Study Population regarding echocardiographic parameters, surgical data and pathological examination.

\begin{tabular}{lccc}
\hline & Without POAF & POAF & P value \\
\hline \multicolumn{1}{c}{ Preoperative transthoracic echocardiogram } & & \\
\hline LAD (mm) & $40.3 \pm 0.95$ & $42.4 \pm 0.97$ & .12 \\
LAVI (mL/m2) & $27.1 \pm 1.7$ & $32.8 \pm 2.4$ & 0.06 \\
LA-EF (\%) & $54.0 \pm 2.7$ & $45.6 \pm 2.1$ & 0.02 \\
PA-TDI (milliseconds) & $120.8 \pm 1.8$ & $152.1 \pm 3.0$ & $<0.001$ \\
LV-EF (\%) & $60.1 \pm 1.6$ & $58.6 \pm 2.9$ & 0.67 \\
Operative parameters & & & \\
Replacement by metallic valve & 45 & 31 & 0.74 \\
Repair & 8 & 2 & \\
Tissue valve & 13 & 12 & 0.67 \\
Duration of surgery (minutes) & $193.6 \pm 15.8$ & $184.3 \pm 19.0$ & 0.09 \\
Aortic cross clamp time & $62.8 \pm 7.0$ & $91.0 \pm 14.7$ & \\
Histopathologic parameter & & & \\
Amount of RAA fibrosis (\%) & $16.56(5.39)$ & $27.01(11.8)$ & $<0.001$ \\
\hline
\end{tabular}

Table (3) Using Multiple Logistic Regression Analysis to detect independent predictors of PAOF.

\begin{tabular}{lccccc}
\hline & B & SE & WALD & P value & OR (95\% CI) \\
\cline { 2 - 6 } Atrial fibrosis & 4.235 & 0.291 & 220.2 & $<0.001$ & $68.1(39.0-132.6)$ \\
PA-TDI & 2.001 & 0.113 & 331.0 & $<0.001$ & $7.4(5.7-9.4)$ \\
LAEF & -0.177 & 0.043 & 17.5 & $<0.01$ & $0.84(0.78-0.91)$ \\
LAVI & 0.102 & 0.043 & 7.1 & $<0.05$ & $0.90(0.83-0.98)$ \\
Aortic cross clamp time & -0.043 & 0.042 & 1.2 & 0.317 & $0.96(0.89-1.04)$ \\
\hline
\end{tabular}

\section{Discussion}

\subsection{Key findings}

The current study examined the correlation between atrial fibrosis and total atrial conduction time and its relation with development of post-operative atrial fibrillation. The key findings in this study include that patients who had an early POAF had higher amount of fibrosis in their atrial tissue as shown by the sample. In addition, TACT helped in classifying the patients who had high risk for developing POAF after undergoing surgical intervention. Moreover, TACT shows the burden of atrial fibrosis and subsequent remodeling in the atrial tissue.

\subsection{TACT and Post-operative atrial fibrillation}

Earlier studies revealed that PA-TDI interval could predict early onset AF postoperatively.

Measuring PA-TDI interval is quite consistent with low inter-observer variation; therefore, measuring it twice is applicable and can be used with confidence in evaluating the risk of the post-operative atrial fibrillation [6].

Roshanali et al. was the first study to examine and confirm that TACT could be used to detect the risk of POAF undergoing $\mathrm{CABG}$. A cut-off point of 121 milliseconds was defined for predicting POAF (sensitivity 99\%; specificity $93.8 \%$ ). According the cut-off point of the previous study, we considered an optimal cut-off point of 132 milliseconds (sensitivity $99 \%$, specificity $85 \%$ ) using phi correlation coefficient [7].

In our study LA-ejection, fraction and PATDI interval were the only two independent parameters, which are associated with early POAF Table (1). In addition, multivariate Analysis showed that PA-TDI interval was the echocardiographic variable that was able to predict post-operative atrial fibrillation Table (3) [7].

\subsection{Correlation of TACT and Structural Atrial Remodeling}

Atrial fibrosis is considered the precursor for initiation and perpetuation of atrial fibrillation [8].

Oakes et al. showed that amount of fibrosis detected by magnetic resonance imaging delayed-enhancement which is considered to be a noninvasive tool for assessment of left atrial fibrosis within myocardial tissue in AF patient and could evaluate the disease progression.20 However, its use is limited due to practical limitation [9].

Age is considered to be an established risk factor for $\mathrm{AF}$ and earlier studies showed a positive correlation between RAA fibrosis and the patient undergoing open heart surgery age [10]. 
Both Platonov et al. and the current could not detect such a correlation [11].

\section{Limitation}

Small sample size and being a single center experience are the main limitation of this study

\section{Conclusion}

PA-TDI is an emerging parameter that would be helpful in risk stratification of patients undergoing mitral valve replacement and further investigation of this novel parameter in different population would be beneficial in assessing its ability of predicting POAF.

\section{References}

[1] G.H. Almassi, T.L. Schowalter, A.C. Nicolosi, A.Y. Aggarwal, T.E. Moritz, W.G. Henderson, R.L. Tarazi, A.L. Shroyer, G.K. Sethi, F.L. Grover and K.E. Hammermeister, Atrial fibrillation after cardiac surgery: a major morbid event. Ann Surg, Vol.226, PP.501-513, 1997.

[2] S.F. Aranki, D.P. Shaw, D.H. Adams, R.J. Rizzo, G.S. Couper, M.N. VanderVliet, J.J. Collins, L.H. Cohn, and H.R. Burstin, Predictors of atrial fibrillation after coronary artery surgery Current trends and impact on hospital resources. Circulation, Vol.94, PP.390 -397, 1996.

[3] G.K. Mariscalco and K.G. Engström, Atrial fibrillation after cardiac surgery: Risk factors and their temporal relationship in prophylactic drug strategy decision. Int $\mathbf{J}$ Cardiol, Vol.129, PP. 354-362, 2007.

[4] E.J. Orlowska-Baranowska, R.P. Baranowski, P.O. Michalek, P.I. Hoffman, T.O. Rywik and I.L. Rawczylska-Englert, Prediction of paroxysmal Atrial fibrillation after aortic valve replacement in patients with aortic stenosis: identification of potential risk factors. J Heart Valve Dis, Vol.12, PP.136-141, 2003.

[5] F.K. Roshanali, M.H. Mandegar, M.A. Yousefnia, F.L. Alaeddini and B.T. Saidi, Prevention of atrial fibrillation after coronary artery bypass grafting via atrial electromechanical interval and use of amiodarone prophylaxis. Interact Cardiovasc Thorac Surg, Vol.8(4), PP.421425, 2009.

[6] K.L. Merckx, C.B. De Vos, A.L. Palmans, J.I. Habets, E.C. Cherlex, H.J. Crijns and R.G. Tieleman, Atrial activation time determined by transthoracic Doppler tissue Imaging can be used as an estimate of the total duration of atiral electrical activation. J Am Soc Echocardiogr, Vol.18, PP.940944, 2005.

[7] F.L. Roshanali, M.H. Mandegar, M.A. Yousefnia, F.k. Alaeddini and B.L. Saidi, Prevention of atrial fibrillation after coronary artery bypass grafting via atrial electromechanical interval and use of amiodarone prophylaxis. Interact Cardiovasc Thorax Surg, Vol.8, PP.421425, 2009.

[8] B.R. Maessen, J.M. Nijs, J.L. Maessen, M.P. Allessie and U.O. Schotten, Postoperative atrial fibrillation: A maze of mechanisms. Europace Vol.14, PP.159174, 2012.

[9] R.S. Oakes, T.J. Badger, E.G. Kholmovoski, N. Akoum, N.S. Burgon, E.N. Fish, J.J. Blauer, S.N. Rao, E.V. DiBella, N.M. Segerson, M. Daccarett, J. Windfelder, C.J. McGann, D. Parker, R.S. MacLeod and N.F. Marrouch, Detection and quantification of left atrial structural remodeling with delayed enhancement magnetic resonance imaging in patients with atrial fibrillation. Circulation, Vol. 13, PP.1758-1767, 2009.

[10]F.L. Gramley, J.R. Lorenzen, C.D. Knackstedt, O.R. Rana, E.P. Saygili, D.T. Frechen, S.T. Stanzel, F.U. Pezzella, E.O. Koellensperger, C.Y. Weiss, T.U. Munzel and P.C. Schauerte, Age related atrial fibrosis. Age, Vol.1, PP.27-38, 2009.

[11]P.G. Platonov, L.B. Mitrofanova, V.R. Orshanskaya, and S.Y. Ho, Structural abnormalities in atrial walls are associated with presence and persistency of atrial fibrillation but not with age. Journal of the American College of Cardiology, Vol.58 (21), PP.2225-2232, 2011. 